\title{
CHRONIC CARPAL TUNNEL SYNDROME: RESULTS OF CARPAL TUNNEL RELEASE
}

\author{
Gulzar Saeed Ahmed
}

\begin{abstract}
BACKGROUND/OBJECTIVE: Carpal tunnel syndrome (CTS) is the commonest entrapment neuropathy for which several conservative and surgical treatment options are available. In majority of cases the cause of CTS is unknown (idiopathic CTS), however, there are numerous medical conditions associated with CTS. Surgery for CTS is one of the most often performed procedures. The purpose of this study was to evaluate the clinical outcome of surgical treatment of chronic carpal tunnel syndrome.

METHODS: Thirty patients with chronic CTS were selected for open carpal tunnel release. Twenty patients had bilateral disease. Thirty hands had thenar muscle atrophy. Fifty hands of thirty patients were operated from May 1997 to December 2002.

RESULTS: Sensation improved within three months in all patients. Nocturnal pain disappeared within first week after surgery. Grip strength improved gradually even up to two years after surgery. All of thirty hands with thenar muscle atrophy showed symptomatic relief, but recovery of the wasted muscle in term of regaining bulk was very slow in most of cases. Only ten hands showed complete recovery of bulk of thenar muscles in three years time.

CONCLUSION: Surgery is an excellent treatment option in chronic CTS in terms of symptomatic relief.Wasted thenar muscles recover slowly in fraction of cases.
\end{abstract}

KEY WORDS: Carpal Tunnel Syndrome. Release. Thenar. Surgery.

\section{INTRODUCTION}

Carpal tunnel syndrome (CTS) is the most common entrapment neuropathy. ${ }^{1}$ Surgery for CTS is one of the most often performed procedures. In the United States, more than 350,000 carpal tunnel release procedures are performed annually. ${ }^{2}$ Any condition that reduces the size of the carpal tunnel or increases the volume of its content may cause compression of the median nerve. In majority of cases the cause of CTS is unknown, called as idiopathic CTS. However, there are numerous medical conditions associated with CTS, such as diabetes mellitus, thyroid disease, rheumatoid arthritis and pregnancy. ${ }^{3}$ The symptoms of CTS include pain, par aesthesia and hypoesthesia in the hand, in the area innervated by the median nerve, and often occurs or worsens during the night or early morning, waking the patient up. There may also be associated loss of sensibility and strength. The clinical diagnosis of CTS can be confirmed by electro diagnostic studies, which have been found to be highly sensitive (49\% to $84 \%$ ) and specific (95\% or greater). ${ }^{4}$ Role of magnetic resonance imaging has also been identified in diagnosis of CTS. MR imaging reliably depicts normal carpal tunnel anatomy. It can also identify pathologic nerve compression and mass lesions, such as ganglion cyst that compress nerves. ${ }^{5}$ For the treatment of CTS, several conservative and surgical options are available. The most commonly used conservative treatment options are wrist splinting, injection of corticosteroids into the carpal tunnel, non-steroidal anti-inflammatory drugs (NSAIDs), systemic steroids, pyridoxine (Vitamin $\mathrm{B}_{6}$ ) and diuretics. ${ }^{6-7}$ However, there is only limited evidence of the efficacy of any of these conservative treatment options. ${ }^{8}$ Surgical treatment options consist of sectioning the transverse carpal ligament, resulting in an increased canal volume and/or widening the bony carpal arch, thereby reducing the pressure on the median nerve. There are several surgical techniques available, e.g. open carpal tunnel release(OCTR), adding epineurotomy or internal neurolysis to OCTR, or endoscopic carpal tunnel release, but standard OCTR seems to offer similar relief from CTS symptoms in the short and long-term. ${ }^{9}$ The purpose of this study was to evaluate the clinical outcome of surgical treatment of chronic carpal tunnel syndrome.

\section{PATIENTS AND METHODS}

Fifty hands of thirty patients were operated for carpal tunnel release at Liaquat Medical College Hospital Jamshoro, Isra University Hospital Hyderabad and three private hospitals in Hyderabad, Sindh - Pakistan from May 1997 to December 2002. Out of these thirty patients, twenty were female. The age range was between 30 to 60 years. The duration of symptoms was 
between 9 to 20 months. Four patients had diabetes mellitus and one had hyperthyroidism. Most of the patients presented with burning and numbness of hand, waking the patient in the middle of night. Fifteen patients had weakness in hand and complained objects falling from the hand. On physical examination, acute flexion of wrist for 60 seconds increased par aesthesia in the distribution of median nerve in hand in majority of patients. Phalen test - a nerve conduction study for upper limb was performed in all patients. Twenty five patients with abnormal study showing evidence of compression of median nerve at wrist were operated. Five patients with normal nerve conduction study but positive clinical signs were also operated. Out of ten male patients, six were Labourers and four were Office Workers. Out of twenty female patients, fifteen were housewives and five were Office Workers. Three male patients with unilateral disease had swelling on the volar aspect of the involved wrist clinically consistent with ganglion. Six female patients with bilateral disease had rheumatoid arthritis. Five patients received injection hydrocortisone into the carpal tunnel on three occasions with no relief. Sign and symptoms were persistent and progressive in all patients. Thirty hands had thenar muscle atrophy. All the patients were thoroughly examined to rule out nerve compression caused by cervical disc herniation and thoracic outlet obstruction and median nerve compression proximally in forearm and elbow. Surgical release was performed under general anesthesia and tourniquet was applied in the arm. First generation cephalosporin was injected intravenously for prophylaxis at the time of induction of anesthesia followed by two doses six hourly. Patients with bilateral disease were operated on both extremities under same anesthesia.

Procedure: ${ }^{10}$ A curved incision was made ulnar to and paralleling the thenar crease. The incision was extended proximally to the flexion crease of the wrist. The incision was angled to the ulnar side of the wrist to avoid crossing the flexor crease at right angle and also to avoid cutting the palmer sensory branch of the median nerve that lays in the interval between palmaris longus and the flexor carpi radialis tendon. After incising and reflecting the skin and subcutaneous tissue transverse carpal ligament was divided along its ulnar border to avoid damage to the median nerve and its recurrent branch. Ganglion was excised in three patients and synovectomy was performed in eight out of twelve hands with rheumatoid arthritis. Only the skin was closed and drain was placed. Dressing and volar splint were applied. Drain was removed after forty-eight hours. Stitches were removed after 14 days; splint was maintained for another two to three weeks.

\section{RESULTS}

Fifty hands of thirty patients were operated for carpal tunnel release. Superficial palmer branch of median nerve was accidentally divided in one hand (2\%). No attempt was made to repair the damaged nerve. There were two superficial infections (4\%). Those were treated with antibiotics according to culture and sensitivity. Post operative pain was severe in eleven hands (22\%), of which eight hands were those in which synovectomy was performed and in three hands from which ganglion was excised. These patients required analgesics for six to eight days after surgery (Figure I). Sensation improved within three months in all patients. Nocturnal pain disappeared within first week after surgery. Grip strength improved gradually even up to two years after surgery in majority of patients. All of thirty hands with thenar muscle atrophy showed symptomatic relief, but recovery of the wasted muscle in term of regaining bulk was very slow in most of cases, only ten hands showed complete recovery of bulk of thenar muscles in three years time. Out of thirty patients, twenty four were satisfied with results (Figure II). Six female patients with rheumatoid arthritis were not satisfied as mild pain in wrist persisted and there was no remarkable improvement in grip strength three years after surgery.

\section{FIGURE I: COMPLICATIONS $(n=50)$}

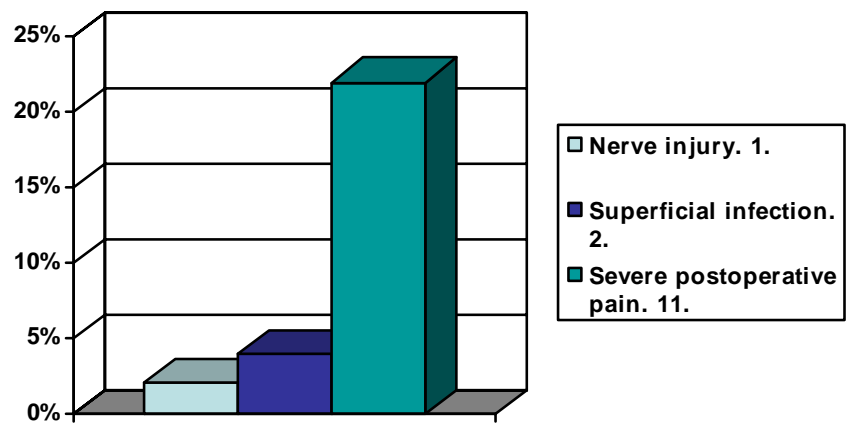

\section{FIGURE II: PATIENTS OPINION REGARDING SURGICAL OUTCOME}

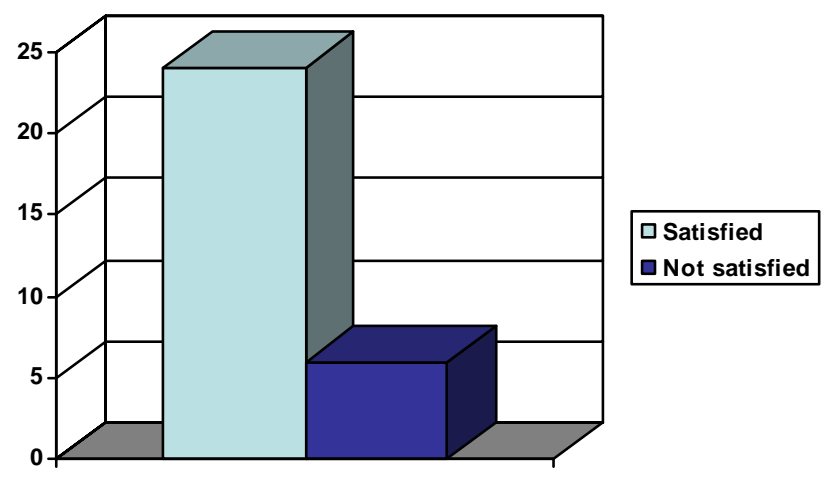




\section{DISCUSSION}

In this study, $66 \%$ of the patients were females. Majority of patients with CTS is female population in most of the published studies. Shabir $\mathrm{M}^{11}$ has shown female to male ratio of $7: 1$ and age range between 30 to 50 years which is comparable with age range of 30 to 60 years in this study. In another study, CTS was reported four times more common in women. ${ }^{12}$ Nerve conduction study was performed in all symptomatic patients. Five out of thirty patients had normal report. Symptomatic relief was seen in all patients after surgery. Nerve conduction study is gold standard for diagnosis of CTS but negative study does not mean that syndrome does not exist. It is said that release operation can be performed in presence of symptoms and it is not necessary to perform nerve conduction study in all patients. ${ }^{13}$ Conservative treatment options of the CTS are immobilization of the wrist, physiotherapy, drug therapy and steroid injections into carpal tunnel. These measures are effective for short time but not in long term follow up in most of the patients. The most common treatment in the carpal tunnel syndrome is surgery, which consists of division of the transverse carpal ligament. ${ }^{14}$ There is no agreement on the choice of initial treatment for CTS. American Academy of Neurology advises non-invasive treatment first and using invasive steroid injection or open carpal tunnel release only if non-invasive treatment is ineffective. ${ }^{15}$ Advocates of early surgery point out that conservative therapy offers only temporary symptomatic relief. They argue that unnecessary delay in surgery can cause further damage to the median nerve. ${ }^{16}$ Advocates of initial conservative management of CTS however, refer to the potential benefits and safety of conservative treatment options and the potential complications of surgery. ${ }^{17}$ All the patients included in this study had chronic symptoms (9 to 20 months) and thirty hands had thenar muscle atrophy. Open carpal tunnel release was treatment of choice in all patients. Results of surgical treatment in this study, in terms of improvement of sensation, disappearance of night pain and improvement of grip strength are comparable to those reported in literature. ${ }^{14}$

\section{CONCLUSION}

Surgery is essential and rewarding in providing symptomatic relief and preventing further damage to median nerve in chronic carpal tunnel syndrome. Once, thenar muscle wasting sets in, it is difficult to regain muscle bulk after surgery.

\section{REFERENCES}

1. Practice parameters for carpal tunnel syndrome (Summary Statement). Report of the Quality Standards Subcommittee of the American Academy of Neurology. Neurology. 1993; 43(11):2406-9.

2. Owings MF, Kozak LJ. Ambulatory and inpatient procedures in the United States, 1996. Vital Health Stat. 1998 ; (139):1-119.

3. Dawson DM. Entrapment neuropathies of the upper extremities. New England J Med. 1993; 329 (27):2013-8.

4. Practice parameters for electro diagnostic studies in carpal tunnel syndrome: summary statement. American Association of Electro diagnostic Medicine, American Academy of Neurology, American Academy of Physical Medicine and Rehabilitation. Muscle Nerve. 1993; 16: 1390-1.

5. Jarvik JG, Yuen E, Kliot M. Diagnosis of carpal tunnel syndrome: Electro diagnostic and MR imaging evaluation. Neuroimaging Clin N Am. 2004; 14(1): 93-102.

6. Pal B, Morris J, Keenan J, et al. Management of idiopathic carpal tunnel syndrome. A survey of rheumatologists' practice and proposed guidelines. $\mathrm{Br}$ J Rheumatol. 1997; 36:1328-30.

7. Scholten RJPM, de Krom MC, Bertelsmann FW, et al. Variation in the treatment of carpal tunnel syndrome. Muscle Nerve. 1997; 20:1334-5.

8. Gerritsen AA, de Krom MC, Struijs MA, et al. Conservative treatment options for carpal tunnel syndrome: a systematic review of randomized clinical trials. J Neurol. 2002; 249(3):272-80.

9. Gerritsen AAM, Uitdehaag BMJ, van Geldere D, et al. Systematic review of randomized clinical trials of surgical treatment for carpal tunnel syndrome. Br J Surg. 2001; 88:1285-95.

10. Phillip E, Wright II. Carpal tunnel and ulnar tunnel syndromes and stenosing tenosynovitis. In: Crenshaw AH, ed. Campbell's operative orthopaedics. Vol 5, 8th edition. St. Louis: Mosby; 1992: Pp. 3435-45.

11. Shabbir M. Surgical treatment of Carpal Tunnel Syndrome. J Postgrad Med Inst. 2004; 18(1):2932.

12. Niazi PHK, Hussain S. Carpal Tunnel Syndrome. Pak Armed Forces Med J. 1996; 46(2):76-9.

13. Finsen $V$, Russwurm $H$. Neurophysiology not required before surgery for typical carpal tunnel syndrome. J Hand Surg [Br]. 2001; 26(1):61-4.

14. Zyluk A, Strychar J. Results of the treatment of 
carpal tunnel syndrome-a review. Chir Narzadow Ruchu Ortop Pol. 2005; 70(6):439-45.

15. Practice parameter for carpal tunnel syndrome (summary statement). Report of the Quality Standards Subcommittee of the American Academy of Neurology. Neurology. 1993; 43:2406-9.
16. Wilson JR, Sumner AJ. Immediate surgery is the treatment of choice for carpal tunnel syndrome. Muscle Nerve. 1995; 18:660-2.

17. Johnson EW. Should immediate surgery be done for carpal tunnel syndrome? - No! Muscle Nerve. 1995; 18:658-9.

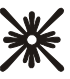

AUTHOR AFFILIATION:

Dr. Gulzar Saeed Ahmed (Corresponding Author)

Associate Professor, Orthopedic Surgery

Isra University Hyderabad, Sindh - Pakistan.

Email: gulzarsaeed@yahoo.com 